\title{
A Successful Chemoembolization of a Retroperitoneal Soft Tissue Sarcoma: A Case Report
}

\author{
Waldec Jorge David Filho ${ }^{a, b} \quad$ Angel Ayumi Tome Uchiyama ${ }^{a}$ \\ Catarina Marchon ${ }^{a}$ Letícia Maria Duarte Lopes ${ }^{b}$ \\ Ana Luísa de Castro Baccarin ${ }^{b}$ Bruna de Fina ${ }^{c}$ \\ Ricardo Virgílio dos Santos ${ }^{c}$ Francisco Leonardo Galastric ${ }^{c}$ \\ a'Oncology, Hospital Alemão Oswaldo Cruz, São Paulo, Brazil; boncology, Instituto Brasileiro \\ de Controle do Câncer, São Paulo, Brazil; ' Vascular, Instituto Brasileiro de Controle do \\ Câncer, São Paulo, Brazil
}

\author{
Keywords \\ Chemoembolization Retroperitoneal sarcoma
}

\begin{abstract}
Retroperitoneal sarcomas are rare entities. They have a tendency of growing slowly, rendering the patient apparently healthy for long periods of time, before diagnosis. Besides, they have a worse prognosis than sarcomas arising in extremities, with a higher local recurrence rate and lower 5-year survival rate. We describe a case of a 71-year-old male patient, who had a very well succeeded treatment of a large retroperitoneal sarcoma with the combination of chemoembolization, systemic chemotherapy, surgery, and radiation therapy. Initially, it was noticed in an incidental way he had a large retroperitoneal mass $(15 \mathrm{~cm} \times 10 \mathrm{~cm} \times 9.2 \mathrm{~cm})$ through magnetic resonance, when he was 63 . The case was considered inoperable by the treating physicians. After neoadjuvant therapy, the residual tumor could be completely excised by the responsible surgeon. With a follow-up of $>5$ years, since the end of treatment, the patient remains in complete remission and, probably, cured from his illness. Large retroperitoneal sarcomas are still a great challenge for oncologists. According to the medical literature, chemoembolization can benefit some patients, but most of them in a palliative setting. In our report, we believe its contribution was critical for a great outcome. In selected cases, it is possible this procedure may be an additional therapeutic modality, as part of a multidisciplinary approach.
\end{abstract}




\section{Introduction}

Soft tissue sarcomas (STS) encompass a heterogeneous group of diseases that arise in any part of the human body, even if their initial presentation predominates in extremities. Although there are dozens of subtypes, the principles of treatment are mostly the same. Surgery is still the main therapeutic modality. It can be used to remove primary lesions, as well as secondary ones, in some cases. Radiotherapy is generally used to improve local control. Besides the external modality, intrabeam has emerged as an excellent option [1]. Chemotherapy may be used in scenario adjuvant, but its principal usefulness is in metastatic disease, in a palliative setting. Other modalities of treatment, like immunotherapy or target therapy, are barely indicated.

Retroperitoneal STS is a rare malignancy, occurring in $0.5-1$ per 100,000 inhabitants [2]. It has a worse prognosis than sarcomas arising in the extremities, with a higher local recurrence rate and lower 5-year survival rate. Some studies have showed a median survival of 72 months for patients with nonmetastatic disease, 28 months for those with local recurrence, and 10 months for those with metastatic disease [3]. The rarity of these tumors and the complexity of their treatment require multidisciplinary management in specialized centers to improve oncologic and clinical outcomes [4]. Surgery, with complete excision of the tumor, is the most important component of treatment and an independent prognostic variable for overall survival (OS) [5, 6].

Patients should be stimulated to participate in clinical trials whenever there is a condition in which the therapeutic approach is not well defined. But, not always physicians have an available study for inclusion of such patients. For instance, how to deal with a large inoperable retroperitoneal tumor in an otherwise healthy man? The role of embolization (alone or in combination with a cytostatic drug) as part of a therapeutic strategy is poorly defined in such a situation.

\section{Case Report/Case Presentation}

We describe a case of a 71-year-old male patient, who had a very well succeeded treatment of a large retroperitoneal sarcoma with the combination of chemoembolization, systemic chemotherapy, surgery, and radiation therapy. Initially, it was noticed in an incidental way he had a large retroperitoneal mass $(15 \mathrm{~cm} \times 10 \mathrm{~cm} \times 9.2 \mathrm{~cm})$ through MRI scan, when he was 63 . In December 2013, he underwent an adequate surgery, and the tumor was completely excised. The corresponding histological exam in conjunction with immunohistochemistry analysis revealed a pleomorphic undifferentiated sarcoma. In May 2014, during his follow-up, a CT scan showed a recurrent lesion, measuring $20 \mathrm{~cm}$ in its largest diameter. On that occasion, he complained of fatigue, back pain, and weight loss (about $30 \mathrm{~kg}$ during the last 6 months). The staging was performed with a positron emission tomography (PET) scan that reveals no other lesion, but the recurrent one (SUV = 14.5). It was said it could not be removed surgically, and besides there was invasion vascular of the right iliac artery. Hence, 3 cycles of neoadjuvant chemotherapy with the combination of epirubicin $\left(60 \mathrm{mg} / \mathrm{m}^{2} /\right.$ day for 2 consecutive days $)$ and ifosfamide ( $1.8 \mathrm{~g} / \mathrm{m}^{2} /$ day for 5 consecutive days) with mesna for uroprotection, plus granulocytic-colony stimulating factor, was planned in order to minimize the risk of a febrile neutropenia. Such treatment was initiated on July 1, 2014. In September 2014, after the third cycle, a new MRI scan was performed to evaluate the therapeutic response; no significant tumor reduction was observed. After a tumor board discussion, it was decided to try tumor reduction by a transcatheter arterial chemoembolization (TACE). Under general anesthesia, the patient underwent a femoral catheterization, and one vial of DC bead 100-300 microspheres carried 


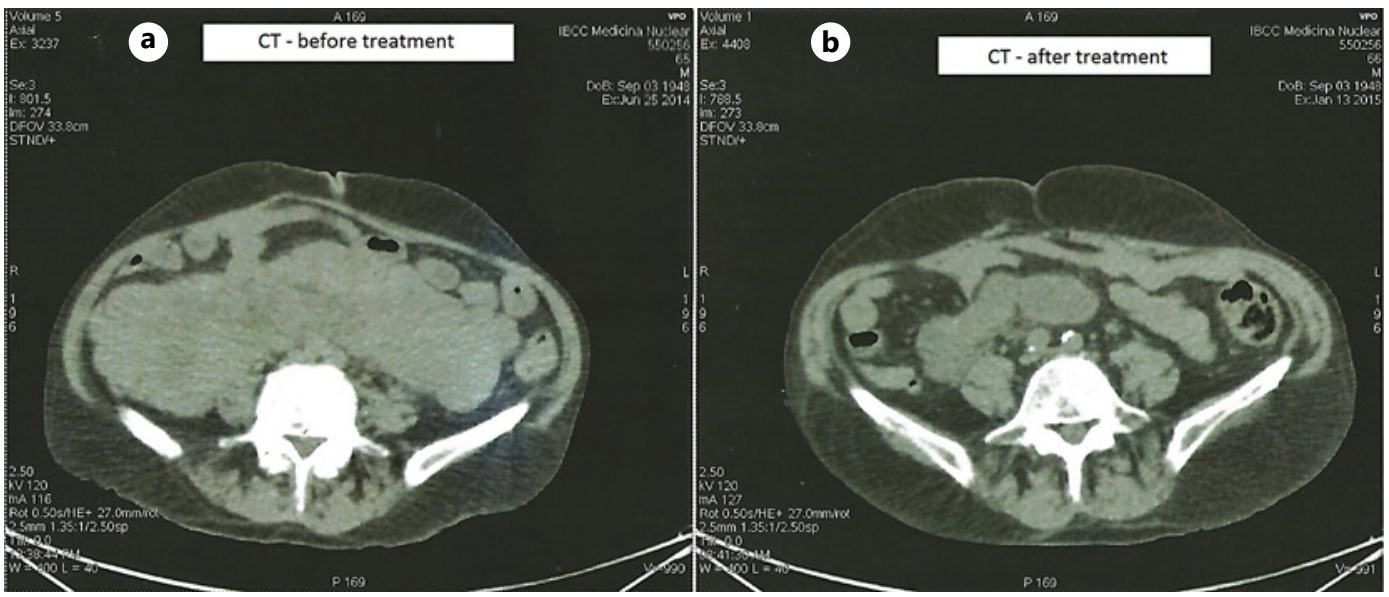

Fig. 1. a Large retroperitoneal tumor before treatment. b Residual tumor after treatment.

with $75 \mathrm{mg}$ of doxorubin was selectively injected into the responsible arteries for the tumor nutrition. He experienced a clinical benefit (decrease of back pain), and a similar second TACE was performed on December 4, 2014. No significant toxicity was noticed in both procedures. In January 2015, a new PET-CT showed a significant tumor reduction with the treatment: from 20 $\mathrm{cm} \times 17 \mathrm{~cm} \times 5.5 \mathrm{~cm}$ to $11.4 \mathrm{~cm} \times 9.5 \mathrm{~cm} \times 4.6 \mathrm{~cm}$, as seen in CT scan (shown in Fig. 1), as well as a disappearance in its metabolic activity (shown in Fig. 2).

On April 4, 2015, the patient underwent an adequate surgery; the whole residual lesion could be removed. After that, he received 50 Gy of intensity-modulated radiation therapy, delivered at tumor bed, given as 1 session per day ( 2 Gy at each session), 5 days a week for 5 consecutive weeks, from August 13 to September 27, 2015. Since then, the patient is healthy and free of symptoms; he was seen for the last time in March 2021.

\section{Discussion/Conclusion}

In 2014, we evaluated a man carrying a large recurrent tumor in his abdominal cavity, which could not be surgically resected up front. It is known that an incomplete tumor excision is related to a lower survival rate [6]. In this case, the antineoplastic chemotherapy had been ineffective, and radiotherapy fields were too large to be considered. Therefore, we pursed a multidisciplinary approach to propose a therapeutic plan that could improve oncological and clinical result.

Could chemoembolization be a reasonable alternative? As it was mentioned above, the role of TACE in the treatment of STS is poorly defined. The results in the medical literature from small series of patients are controversial, but some benefit can be found. TACE is a treatment that blocks blood supplies to tissues or an organ, by placing a synthetic material, called embolic agents, into a blood vessel that feeds the tumor. It may also deliver cytostatic drugs to the tumor with a higher concentration and a possible longer length of stay of the chemotherapeutic agent inside the tumor. The embolization procedure cuts off tumor blood nutrition, attempting to provoke lesion ischemic, which acts synergistically with the chemotherapy cytotoxic effect, enhancing tumor necrosis [7].

Several studies have reported that tumor necrosis induced by chemotherapy correlates with better prognosis in osteosarcoma [8]. It is possible that this benefit can be seen with TACE, as well. In a study conducted by Zhang et al. [9], the usage of TACE before limb salvage 


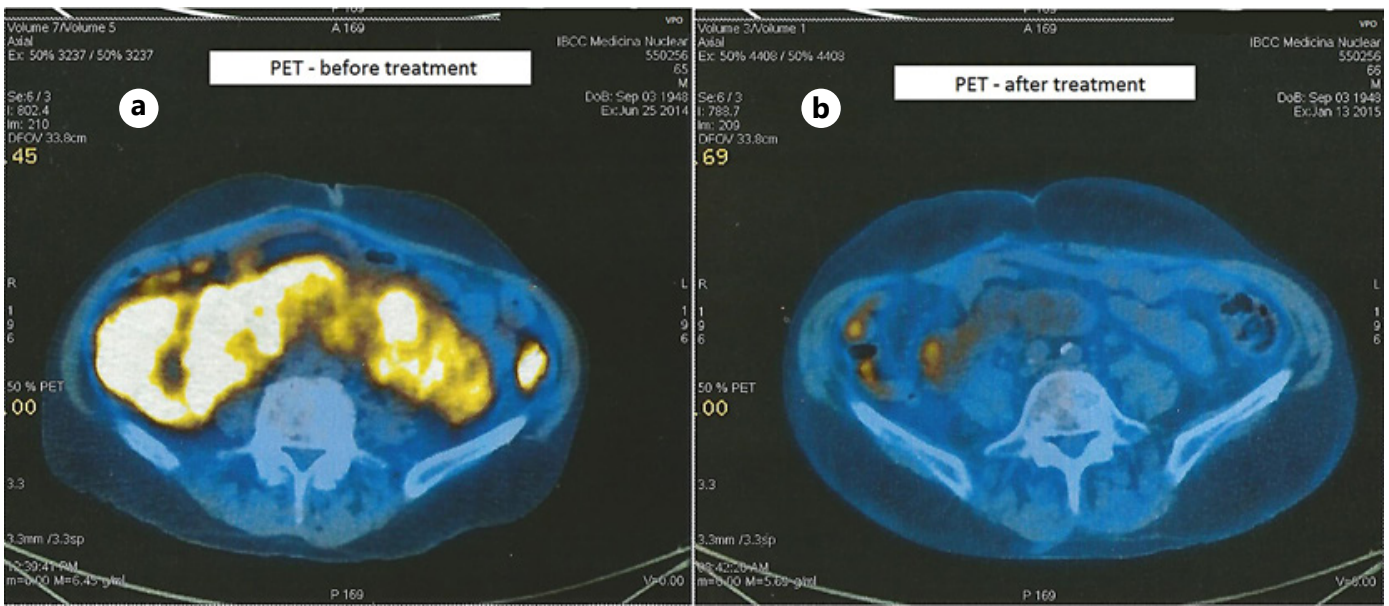

Fig. 2. a Metabolic activity of the tumor before treatment. b Disappearance of metabolic activity after treatment.

surgery was evaluated in 47 patients with osteosarcoma of the lower extremity, resulting in a substantial reduction or complete disappearance of tumor in all patients. According to them, the percentage of tumor necrosis induced by treatment ranged from $70.2 \%$ to $94.2 \%$ (average, 82.9\%).

In another correlated publication, Jiang et al. [10] used TACE in the treatment of 39 patients with unresectable bone and STS, reporting this therapeutic modality was able to lessen cancer pain and increase interval of disease progression. They concluded TACE was an efficient approach, providing an OS rate of $71.5 \%$ and $32.5 \%$ in 1 year and 3 years, respectively.

Similarly, Nagata et al. [11] performed TACE in 10 patients with primary osseous and STS. In 8 cases $(80 \%)$, there was as a significant tumor necrosis; 8 out of 9 patients with painful primary tumors experienced pain control 1 week after the procedure. The median survival was 18 months, and 1-year survival rate was 38.1\%.

Pierce etal. [12] also corroborated that TACE is safe and can have a role in the management of patients with hepatic sarcomas, who are not candidates for curative surgeries. They analyzed OS of 28 patients with unresectable tumors, either primary or metastatic ones, retrospectively. According to their findings, most of them (61\%) obtained a successful outcome, with mild or no treatment-related side effects, and with an average OS of 26.7 months, assessed by Kaplan-Meier analysis.

Additionally, in a case series, 3 patients with giant highly vascular thoracic sarcomas were treated with preoperative embolization followed by surgery. With this technique, a reduction in tumor size ranging from $20 \%$ to $32 \%$ was observed, which provided a complete mass resection in all cases [13]. The most important data from the medical literature about TACE in cancer are listed in Table 1.

What can be said about TACE in patients who previously underwent chemotherapy? Ni et al. [14] performed this therapeutic modality in 10 patients with unresectable STS refractory to conventional systemic chemotherapy. They illustrated effectiveness and safety with TACE therapy in this population, improving OS. The objective response rate was $30 \%$, the median progression-free survival was 9.5 months (range 2-15 months), and the median survival time was 21 months (range 11-30 months). OS rate at 1 and 2 years was $90 \%$ and $30 \%$, respectively. There were no procedure-related mortalities or serious adverse events during the follow-up period. The postembolization syndrome caused by TACE 


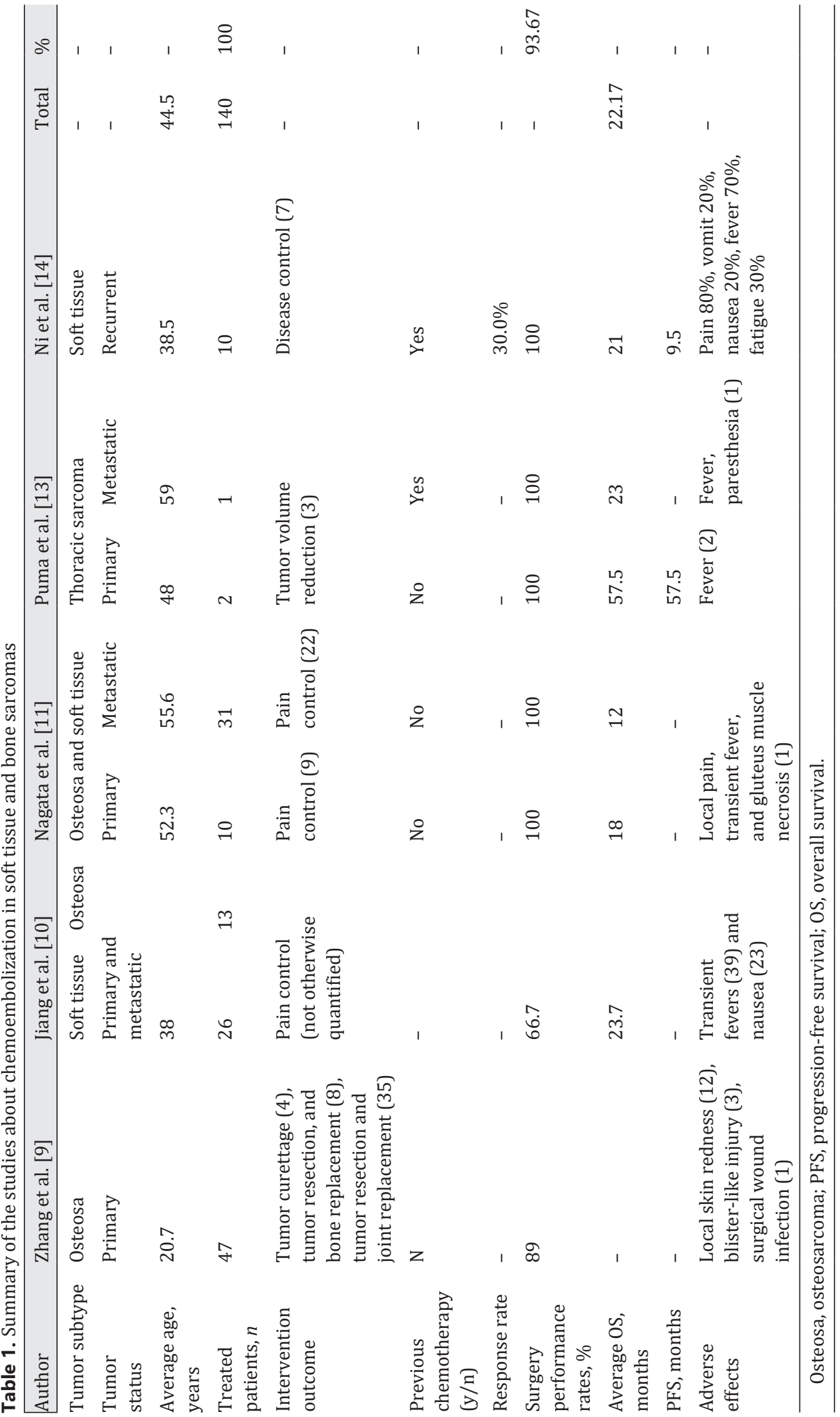


treatment, such as localized pain (80\%), vomiting (20\%), nausea (20\%), fever (70\%), and fatigue $(30 \%)$, was the most common adverse events, being overcome within a few days of the procedure.

We must emphasize that according to some previous studies, the median survival time of STS patients who underwent systemic chemotherapy ranged from 14 to 17 months [15, 16]. Our patient has, so far, a long relapse-free survival. Besides, the patient we have described did not experience pain as a relevant symptom, as it could have been expected. Nevertheless, a significant tumor shrinkage was noticed, which enabled a complete resection of the tumor mass. We believe the main therapeutic modality responsible for that successful treatment was the chemoembolization. The studies cited above showed that TACE was effective and safe for patients with STS. Therefore, this procedure can be considered as a treatment option for unresectable STS refractory to systemic chemotherapy.

In conclusion, we can say that large retroperitoneal STS are still a great challenge for oncologists. These tumors tend to grow silently and can reach dimensions too big to be removed properly. Hence, recurrences are frequent, and the challenge becomes even greater. Thus, in selected cases, it is possible that TACE might be an additional therapeutic modality for those patients. It would be recommendable that cancer centers join with each other in order to elaborate a protocol for such a proposition.

\section{Acknowledgments}

The authors are thankful to medical and nursing staff that collaborated with this case, as well as the patient that allowed to be reported.

\section{Statement of Ethics}

Case reports do not require approval by the Ethics Committee of Brazilian hospitals. Written informed consent was obtained from the patient for publication of this case report and any accompanying images.

\section{Conflict of Interest Statement}

The authors have no conflicts of interest to be declared.

\section{Funding Sources}

The authors have no funding to be reported.

\section{Author Contributions}

All the authors contributed to manuscript and review. Waldec Jorge David Filho was responsible for writing the Introduction, the Case Report, and the Discussion/Conclusion sections and was a treating physician, as well. Angel Ayumi Tome Uchiyama was responsible for reviewing of the literature and writing the Discussion section, as well. Catarina Marchon was responsible for the construction of Table 1. Letícia Maria Duarte Lopes was responsible

\section{Karger'}


for writing the "Case Report," section as well. Ana Luísa de Castro Baccarin was one of the treating physicians and leaded the tumor board. Bruna de Fina performed the first chemoembolization and collaborated with the Conclusion section. Ricardo Virgílio dos Santos performed the second embolization. Francisco Leonardo Galastri described the chemoembolization and collaborated with the Case Report section.

\section{Data Availability Statement}

The data that support the findings of this report can be found in the patient's file. It is not accessible for public consultation, but such a consultation can be requested.

\section{References}

1 David Filho WJ, Hanriot RM, Di Paula Filho RP, Silva PAIA, Costa MADSB, Yen CT, et al. Retrospective analysis of intraoperative radiation therapy with low-kilovoltage x-rays (IORT-LK) in soft tissue sarcomas (STS). J Clin Oncol. 2020 May;38(15):e23575.

2 Gatta G, Capocaccia R, Botta L, Mallone S, De Angelis R, Ardanaz E, et al. Burden and centralised treatment in Europe of rare tumours: results of RARECAREnet-a population-based study. Lancet Oncol. 2017 Aug;18(8): 1022-39.

3 Lewis JJ, Leung D, Woodruff JM, Brennan MF. Retroperitoneal soft-tissue sarcoma: analysis of 500 patients treated and followed at a single institution. Ann Surg. 1998 Sep;228(3):355-65.

4 Derbel O, Heudel PE, Cropet C, Meeus P, Vaz G, Biron P, et al. Survival impact of centralization and clinical guidelines for soft tissue sarcoma (a prospective and exhaustive population-based cohort). PLoS One. 2017 Feb;12(2):e0158406.

5 Bovalot S, Gronchi A, Hohenberger P, Litiere S, Pollock RE, Raut CP, et al.; Trans-Atlantic RPS Working Group. Management of primary retroperitoneal sarcoma (RPS) in the adult: a consensus approach from the TransAtlantic RPS Working Group. Ann Surg Oncol. 2015 Jan;22(1):256-63.

6 Stoeckle E, Coindre JM, Bonvalot S, Kantor G, Terrier P, Bonichon F, et al. Prognostic factors in retroperitoneal sarcoma: a multivariate analysis of a series of 165 patients of the French Cancer Center Federation Sarcoma Group. Cancer. 2001 Jul;92(2):359-68.

7 Lau WY, Lai EC. Hepatocellular carcinoma: current management and recent advances. Hepatobiliary Pancreat Dis Int. 2008 Jun;7(3):237-57.

8 Davis AM, Bell RS, Goodwin PJ. Prognostic factors in osteosarcoma: a critical review. J Clin Oncol. 1994 Feb; 12(2):423-31.

9 Zhang HJ, Yang JJ, Lu JP, Lai CJ, Sheng J, Li YX, et al. Use of intra-arterial chemotherapy and embolization before limb salvage surgery for osteosarcoma of the lower extremity. Cardiovasc Intervent Radiol. 2009 Jul;32(4): 672-8.

10 Jiang C, Wang J, Wang Y, Zhao J, Zhu Y, Ma X, et al. Treatment outcome following transarterial chemoembolization in advanced bone and soft tissue sarcomas. Cardiovasc Intervent Radiol. 2016 Oct;39(10):1420-8.

11 Nagata Y, Mitsumori M, Okajima K, Mizowaki T, Fujiwara K, Sasai K, et al. Transcatheter arterial embolization for malignant osseous and soft tissue sarcomas. II. Clinical results. Cardiovasc Intervent Radiol. 1998 May; 21(3):208-13.

12 Pierce DB, Johnson GE, Monroe E, Loggers ET, Jones RL, Pollack SM, et al. Safety and efficacy outcomes of embolization in hepatic sarcomas. AJR Am J Roentgenol. 2018 Jan;210(1):175-82.

13 Puma F, Cardini CL, Passalacqua G, Ragusa M. Preoperative embolization in surgical management of giant thoracic sarcomas. Eur J Cardiothorac Surg. 2008 Jan;33(1):127-9.

14 Ni JY, Sun HL, Chen YT, Luo JH, Wang WD, Jiang XY, et al. Drug-eluting bead transarterial chemoembolization in the treatment for unresectable soft tissue sarcoma refractory to systemic chemotherapy: a preliminary evaluation of efficacy and safety. J Cancer Res Clin Oncol. 2018 Jan;144(1):157-63.

15 Judson I, Verweij J, Gelderblom H, Hartmann JT, Schöffski P, Blay J-Y, et al.; European Organisation and Treatment of Cancer Soft Tissue and Bone Sarcoma Group. Doxorubicin alone versus intensified doxorubicin plus ifosfamide for first-line treatment of advanced or metastatic soft-tissue sarcoma: a randomised controlled phase 3 trial. Lancet Oncol. 2014 Apr;15(4):415-23.

16 Ryan CW, Matias C, Agulnik M, Lopez-Pousa A, Williams C, de Alwis DP, et al. A phase II study of tasisulam sodium (LY573636 sodium) as second-line or third-line treatment for patients with unresectable or metastatic soft tissue sarcoma. Invest New Drugs. 2013 Feb;31(1):145-51. 